Mathematical Research Letters 1, 359-367 (1994)

\title{
FLAT ANALYTIC DISCS ATTACHED TO REAL HYPERSURFACES OF FINITE TYPE
}

\author{
Serge Alinhac, M.S. Baouendi, and Linda Preiss Rothschild
}

\section{Introduction}

A smooth analytic disc valued in $\mathbb{C}^{n}$ is a map $A: \bar{\Delta} \rightarrow \mathbb{C}^{n}$, where $\Delta$ denotes the unit disc in $\mathbb{C}$, with $A$ smooth in $\bar{\Delta}$ and holomorphic in $\Delta$. We shall say that the disc $A$ is attached to a manifold $M \subset \mathbb{C}^{n}$ if $A(S) \subset M$, where $S=\partial \Delta$. Similarly, $A$ is partially attached to $M$ if $A(I) \subset M$, where $I \subset S$ is an open arc with $1 \in I$. In [1] the authors proved the following unique continuation result. If $A$ is partially attached to a totally real manifold $M$ and flat at 1, i.e. $A^{(j)}(1)=0, j=1,2, \ldots$, then $A$ is constant. On the other hand, it is well known that if $M$ is a strictly pseudoconvex hypersurface and $A$ is a sufficiently small nonconstant analytic disc attached to $M$, then $A^{\prime}(1) \neq 0$. (See Remark (4.3).) It should be mentioned that starting with the work of Bishop [5] and Lewy [9], analytic discs attached to hypersurfaces and their boundary derivatives have played an important role in local and global CR geometry for such problems as holomorphic extension of CR functions and propagation of holomorphic extension. For recent work see e.g. Trépreau [10], Tumanov [11], and [3].

It has been an open question whether there can exist a smooth nonconstant analytic disc $A$ attached to a hypersurface of finite type [8] in $\mathbb{C}^{2}$ with $A$ flat at 1 . In this paper we first characterize flat discs valued in $\mathbb{C}$ in terms of the Fourier transform of their pullbacks to the real line. (See Boutet de Monvel [4] for a similar approach.) We then use this characterization to construct arbitrarily small discs flat at 1 attached to a large class of hypersurfaces, including any homogeneous hypersurface of odd type.

We conclude the paper with some remarks about sufficient conditions on a hypersurface $M$ which imply that $A^{\prime}(1) \neq 0$ for any smooth analytic disc attached to $M$.

Received January 10, 1994.

The second and third authors were partially supported by National Science Foundation Grant DMS 9203973. 


\section{Smooth and flat functions on the circle}

Let $S$ be the unit circle in the complex plane $\mathbb{C}$ and

$$
C_{*}^{\infty}(S)=\left\{f \in C^{\infty}(S): f(1)=0\right\},
$$

where the functions are complex valued. We now consider the mapping $R: \mathbb{R} \rightarrow S$ given by $R(s)=\frac{s-i}{s+i}$, and first describe the pullback of $C_{*}^{\infty}(S)$ under $R$. Let

$$
\mathcal{E}(\mathbb{R})=\left\{h \in C^{\infty}(\mathbb{R}): s \mapsto h\left(\frac{1}{s}\right) \in C^{\infty}(\mathbb{R}), \quad \lim _{|s| \rightarrow \infty} h(s)=0\right\} .
$$

We consider the map $C_{*}^{\infty}(S) \ni f \mapsto f \circ R \in \mathcal{E}(\mathbb{R})$ and write $h(s)=f\left(\frac{s-i}{s+i}\right)$. Finally, we put

$$
\mathcal{F}(\mathbb{R})=\{\phi: \phi(\sigma)=H(\sigma) \mu(\sigma)+H(-\sigma) \nu(\sigma) \text { with } \mu, \nu \in \mathcal{S}(\mathbb{R}), \sigma \in \mathbb{R}\},
$$

where $\mathcal{S}(\mathbb{R})$ is the Schwartz space of rapidly decreasing functions on $\mathbb{R}$, and $H(\sigma)$ is the Heaviside function, $H(\sigma)=1$ for $\sigma \geq 0$ and $H(\sigma)=0$ for $\sigma<0$.

We have the following:

Proposition 1.4. The mapping $f \mapsto h=f \circ R$ is an algebra isomorphism from $C_{*}^{\infty}(S)$ onto $\mathcal{E}(\mathbb{R})$ with inverse $f\left(e^{i \theta}\right)=h(-\cot (\theta / 2))$. Furthermore, the Fourier transform is an isomorphism between $\mathcal{E}(\mathbb{R})$ and $\mathcal{F}(\mathbb{R})$.

Proof. Note first that since $h(s)=f\left(\frac{s-i}{s+i}\right)$ and $h\left(\frac{1}{s}\right)=f\left(\frac{1-s i}{1+s i}\right)$, it follows that if $f \in C_{*}^{\infty}(S)$, then both $h(s)$ and $h\left(\frac{1}{s}\right)$ are smooth and $h(s)$ vanishes at $\infty$. The inverse $h \mapsto f$ is obtained from $f\left(e^{i \theta}\right)=h\left(i \frac{1+e^{i \theta}}{1-e^{i \theta}}\right)=h(-\cot (\theta / 2))$. For the last statement concerning the Fourier transform, we assume first that $h \in \mathcal{E}(\mathbb{R})$. Let $g(s)=h\left(\frac{1}{s}\right)$. Since $g$ is smooth at 0 and $g(0)=0$, we obtain by differentiation

$$
\left|h^{(j)}(s)\right| \leq \frac{C_{j}}{(1+|s|)^{j+1}}
$$

If $\hat{h}$ denotes the Fourier transform of $h$, it follows from (1.5) that $\frac{d^{k}}{d \sigma^{k}} \sigma^{j} \hat{h}(\sigma)$ is continuous and bounded on $\mathbb{R}$ for $0 \leq k<j<\infty$. To complete the proof that $\hat{h} \in \mathcal{F}(\mathbb{R})$, it remains only to show that

$$
\lim _{\sigma \rightarrow 0^{+}} \hat{h}^{(j)}(\sigma) \quad \text { and } \quad \lim _{\sigma \rightarrow 0^{-}} \hat{h}^{(j)}(\sigma)
$$


exist, for $j=0,1, \ldots$ From the Taylor expansion of $g$ at the origin, there exist unique constants $a_{j}$ such that for any $N>0$,

$$
h(s)=\sum_{j=1}^{N} \frac{a_{j}}{s^{j}}+O\left(\frac{1}{|s|^{N+1}}\right), \quad|s| \rightarrow \infty
$$

For any $k=0,1, \ldots$, let $N=k+1$ in $(1.7)$ and

$$
h_{k}(s)=s^{k} h(s)-\sum_{j=0}^{k} a_{j} s^{k-j}-a_{k+1} P V\left(\frac{1}{s}\right),
$$

where $P V$ denotes principal value. It follows from (1.7) that the Fourier transform of $h_{k}$ is continuous on $\mathbb{R}$, and hence the desired conclusion follows by taking the Fourier transform of (1.8) and observing that each resulting term has right and left limits as $\sigma \rightarrow 0$.

To complete the proof of Proposition 1.4, let $\phi \in \mathcal{F}(\mathbb{R})$ and

$$
h(s)=\frac{1}{2 \pi} \int e^{i \sigma s} \phi(\sigma) d \sigma
$$

Since $h(s)$ is clearly smooth, it suffices to show that

$$
g(s)=\frac{1}{2 \pi} \int e^{i \sigma / s} \phi(\sigma) d \sigma
$$

is smooth at $s=0$, with $g(0)=0$. This can be done by differentiating (1.9) for $s \neq 0$, making use of the identity $e^{i \sigma / s}=\frac{d^{k}}{d \sigma^{k}}\left[e^{i \sigma / s} \frac{s^{k}}{i^{k}}\right]$, and integrations by parts, left to the reader.

We shall say that a smooth function $g$ is flat at a point $p$ if $g$ and all its derivatives vanish at $p$.

Proposition 1.10. Let $f \in C_{*}^{\infty}(S)$. Then $f$ is flat at 1 if and only if $h=f \circ R$ is in $\mathcal{S}(\mathbb{R})$.

Proof. If $f$ is in $C_{*}^{\infty}(S)$ then we have by Proposition $1.3 f\left(e^{i \theta}\right)=$ $h(-\cot (\theta / 2))$. If $g(s)=h(1 / s)$ then

$$
f\left(e^{i \theta}\right)=g(-\tan (\theta / 2))
$$

from which it follows that $f$ is flat at 1 if and only if $g$ is flat at 0 , i.e. $h \in \mathcal{S}(\mathbb{R})$. 


\section{Scalar valued smooth analytic discs}

Let

$$
\mathcal{A}_{*}^{\infty}(\Delta)=C_{*}^{\infty}(\bar{\Delta}) \cap \mathcal{H}(\Delta)
$$

be the space of all scalar valued analytic discs smooth up to the boundary and vanishing at 1 . Denote by $\mathbb{C}^{+}$the upper half complex plane $\Im w>0$ and

$\mathcal{E}\left(\mathbb{C}^{+}\right)=\left\{h \in C^{\infty}\left(\overline{\mathbb{C}^{+}}\right) \cap \mathcal{H}\left(\mathbb{C}^{+}\right): k(w)=h\left(-\frac{1}{w}\right) \in C^{\infty}\left(\overline{\mathbb{C}^{+}}\right), k(0)=0\right\}$.

Also, we write

$$
\mathcal{F}^{+}(\mathbb{R})=\{\phi(\sigma)=H(\sigma) \mu(\sigma): \mu \in \mathcal{S}(\mathbb{R})\},
$$

where $H$ denotes the Heaviside function.

Proposition 2.4. The mapping $f \mapsto h(w)=f\left(\frac{w-i}{w+i}\right)$ is an algebra isomorphism from $\mathcal{A}_{*}^{\infty}(\Delta)$ onto $\mathcal{E}\left(\mathbb{C}^{+}\right)$. Furthermore, the Fourier transform is an isomorphism of $\left.\mathcal{E}\left(\mathbb{C}^{+}\right)\right|_{\mathbb{R}}$, the restriction of $\mathcal{E}\left(\mathbb{C}^{+}\right)$to $\mathbb{R}$, onto $\mathcal{F}^{+}(\mathbb{R})$.

Proof. Let $R(w)=\frac{w-i}{w+i}$ be the Riemann mapping from $\mathbb{C}^{+}$onto $\Delta$. Since $R$ is smooth up to the boundary, it follows that if $f \in \mathcal{A}_{*}^{\infty}(\Delta)$, then $h=$ $f \circ R \in C^{\infty}\left(\overline{\mathbb{C}^{+}}\right) \cap \mathcal{H}\left(\mathbb{C}^{+}\right)$. Similarly, we have $k(w)=h\left(-\frac{1}{w}\right)=f(-R(w))$ is also in $C^{\infty}\left(\overline{\mathbb{C}^{+}}\right) \cap \mathcal{H}\left(\mathbb{C}^{+}\right)$, and $k(0)=f(1)=0$. The inverse map from $\mathcal{E}\left(\mathbb{C}^{+}\right)$into $\mathcal{A}_{*}^{\infty}(\Delta)$ is given by $f(\zeta)=h\left(i \frac{1+\zeta}{1-\zeta}\right)=k\left(-i \frac{1-\zeta}{1+\zeta}\right)$. This proves the first part of the proposition.

To prove the statement about the Fourier transform, we show first that if $\left.h \in \mathcal{E}\left(\mathbb{C}^{+}\right)\right|_{\mathbb{R}}$, then $\hat{h}(\sigma)=0$ for $\sigma<0$. For this, we write

$$
\sigma \hat{h}(\sigma)=-i \int_{\mathbb{R}} e^{-i s \sigma} h^{\prime}(s) d s,
$$

and observe that as in (1.5) we have $\left|h^{\prime}(w)\right| \leq C(1+|w|)^{-2}$ for $w \in \mathbb{C}^{+}$. By deforming the contour of integration in $(2.5)$ into $\mathbb{C}^{+}$we obtain for every $t>0$

$$
\sigma \hat{h}(\sigma)=-i \int_{\Im w=t} e^{i w \sigma} h^{\prime}(w) d w,
$$

which yields

$$
|\sigma \hat{h}(\sigma)| \leq C e^{-t \sigma}, \quad \sigma \in \mathbb{R}
$$


where the constant $C$ is independent of $t$. Letting $t$ arbitrarily large, we obtain from (2.6) that $\hat{h}(\sigma)=0$ for $\sigma<0$. Now by Proposition 1.4, we obtain that $\hat{h} \in \mathcal{F}^{+}(\mathbb{R})$.

Conversely, let $\phi \in \mathcal{F}^{+}(\mathbb{R})$. Then

$$
h(w)=\frac{1}{2 \pi} \int_{0}^{\infty} e^{i \sigma w} \phi(\sigma) d \sigma
$$

is in $C^{\infty}\left(\overline{\mathbb{C}^{+}}\right) \cap \mathcal{H}\left(\mathbb{C}^{+}\right)$. It remains only to prove that $k(w)=h\left(-\frac{1}{w}\right)$ is also in the same space. For this, we note first that since $w \mapsto-\frac{1}{w}$ is a holomorphic mapping of $\mathbb{C}^{+}$onto itself, smooth up to the boundary except at 0 , it follows that $k(w) \in \mathcal{H}\left(\mathbb{C}^{+}\right)$, smooth up to the boundary except perhaps at 0 . To show that $k(w)$ is smooth also at 0 , we write

$$
k(w)=\frac{1}{2 \pi} \int_{0}^{\infty} e^{-i \sigma / w} \phi(\sigma) d \sigma, \quad w \in \mathbb{C}^{+} .
$$

Differentiating (2.7) and integrating by parts as in the end of the proof of Proposition 1.4 , we obtain that $k \in C^{\infty}\left(\overline{\mathbb{C}^{+}}\right)$and $k(0)=0$. This completes the proof of Proposition 2.4.

As a corollary of Proposition 1.10 and 2.4 we obtain the following.

Corollary 2.8. Let $f \in C_{*}^{\infty}(S)$ and $h(s)=f\left(\frac{s-i}{s+i}\right)$ for $s \in \mathbb{R}$. Then the following conditions are equivalent.

(1) $f \in \mathcal{A}_{*}^{\infty}(\Delta)$ and $f$ flat at 1 .

(2) $\left.h \in \mathcal{E}\left(\mathbb{C}^{+}\right)\right|_{\mathbb{R}}$ and $k$ flat at 0 , where $k(w)=h(-1 / w)$.

(3) $h \in \mathcal{S}(\mathbb{R})$ and $\hat{h}(\sigma)=0$ for $\sigma<0$.

(4) $h \in \mathcal{S}(\mathbb{R})$ and $h$ extends continuously to $\mathbb{C}^{+}$as a bounded holomorphic function.

Proof. The equivalence of (1) and (2) is an immediate consequence of Proposition 2.4, together with the identity

$$
f\left(e^{i \theta}\right)=h\left(i \frac{1+e^{i \theta}}{1-e^{i \theta}}\right)=k\left(-i \frac{1-e^{i \theta}}{1+e^{i \theta}}\right)
$$

which shows that the flatness of $f$ at 1 is equivalent to the flatness of $k$ at 0 . The rest of the proof of the corollary follows easily from Proposition 2.4, and is left to the reader. 


\section{Flat discs attached to hypersurfaces in $\mathbb{C}^{2}$}

We consider here a hypersurface $M$ in $\mathbb{C}^{2}$ given by

$$
\Im z_{2}=p\left(z_{1}, \bar{z}_{1}\right)
$$

where

$$
p(u, \bar{u})=\sum_{2 \leq j+k \leq m} a_{j k} u^{j} \bar{u}^{k}, \quad a_{j k}=\bar{a}_{k j},
$$

is a real-valued polynomial defined for $u \in \mathbb{C}$. By a smooth analytic disc attached to $M$ (through the origin) we shall mean a map $A: \bar{\Delta} \rightarrow \mathbb{C}^{2}$, with components in $\mathcal{A}_{*}^{\infty}(\Delta)$, and satisfying $A(S) \subset M$. In addition, we shall say that the $\operatorname{disc} A$ is flat (at 1 ) if the components of $A$ are both flat at 1 .

Theorem 1. Let $M$ be a hypersurface in $\mathbb{C}^{2}$ given by (3.1), and assume that $a_{j k}=0$ whenever $j=k$ in (3.2). Then given $\epsilon>0$ there are nontrivial smooth analytic discs $A$ attached to $M$ through the origin, flat at 1 , and satisfying $|A(\zeta)|<\epsilon$ for all $\zeta \in \bar{\Delta}$.

Note that a smooth analytic disc attached to $M$ through 0 is given by

$$
A(\zeta)=\left(z_{1}(\zeta), z_{2}(\zeta)\right), \quad z_{j}(\zeta) \in \mathcal{A}_{*}^{\infty}(\Delta), \quad j=1,2,
$$

with $\Im z_{2}(\zeta)=p\left(z_{1}(\zeta), \bar{z}_{1}(\zeta)\right)$ for all $\zeta \in S$. Since a holomorphic function on $\Delta$ is determined, up to a constant, by its imaginary part restricted to $S$, it is clear that $A(\zeta)$ is determined by $z_{1}(\zeta)$.

Theorem 1 is an immediate consequence of the following more precise statement.

Theorem 2. Let $M$ be a hypersurface in $\mathbb{C}^{2}$ given by (3.1) with $a_{j k}=0$ whenever $j=k$ in $(3.2)$, and let $\phi \in C_{0}^{\infty}(\mathbb{R})$. Let $h \in \mathcal{S}(\mathbb{R})$ be given by $\hat{h}=\phi$, and put

$$
z_{1}\left(e^{i \theta}\right)=h\left(i \frac{1+e^{i \theta}}{1-e^{i \theta}}\right)
$$

If $\operatorname{supp} \phi \subset[a, b]$ and

$$
0<b-a<\frac{2 a}{m-1}
$$

then the unique analytic disc $A(\zeta)=\left(z_{1}(\zeta), z_{2}(\zeta)\right)$ attached to $M$ through 0 with $z_{1}(\zeta)$ given by (3.4) is flat at 1.

The main step in the proof of Theorem 2 is the following: 
Lemma 3.6. Let $\phi \in C_{0}^{\infty}([a, b])$ and suppose that (3.5) is satisfied. Then if $\hat{h}=\phi$ and $j>k, 2 \leq j+k \leq m$, then $h^{j} \bar{h}^{k}$ is the restriction to $\mathbb{R}$ of a function in $\mathcal{E}\left(\mathbb{C}^{+}\right)$and its Fourier transform has compact support in $(0, \infty)$.

In order to prove Lemma 3.6, we shall use the following version of part of the Paley-Wiener Theorem.

Lemma 3.7. Let $h(w)$ be an entire function. Then $\hat{h}$ is in $C^{\infty}(\mathbb{R})$, with $\operatorname{supp} \hat{h} \subset[a, b], a<b$, if and only if for every $N \in \mathbb{Z}_{+}$,

$$
|h(w)| \leq C_{N}(1+|w|)^{-N} e^{(-a H(t) t-b H(-t) t)}, \quad w \in \mathbb{C},
$$

where $t=\Im w$ and $H$ is the Heaviside function.

Proof of Lemma 3.6. Note that the function $h^{j}(w) \overline{h^{k}(\bar{w})}$ is entire. If $\tilde{h}(w)=\overline{h(\bar{w})}$, then supp $\hat{h} \subset[a, b]$ implies supp $\hat{\tilde{h}} \subset[-b,-a] . \quad$ By (3.8) of Lemma 3.7 we have

$$
\left|h^{j}(w) \tilde{h}^{k}(w)\right| \leq C_{N}^{j+k}(1+|w|)^{-(j+k) N} e^{(-(a j-b k) H(t) t-(b j-a k) H(-t) t)} .
$$

Applying Lemma 3.7 again, we conclude that the support of the Fourier transform of $h^{j} \tilde{h}^{k}$ is contained in $[a j-b k, b j-a k]$. Using now the hypothesis (3.5), we note that $a j-b k>0$ whenever $j>k>0$ and $j+k \leq m$. Hence we may apply Corollary 2.8 to conclude that $h^{j}(s) \tilde{h}^{k}(s)$ satisfies the desired conclusion of Lemma 3.6.

Proof of Theorem 2. First note that since supp $\phi \subset[a, b]$ with $0<a$, it follows from Corollary 2.8 that $z_{1}(\zeta)$ is flat at 1 . Similarly, from Lemma 3.6, we have that if $j>k, j+k \leq m$, the function $s \mapsto g_{j k}(s)=h^{j}(s) \bar{h}^{k}(s)$ extends holomorphically to $\mathbb{C}$ as a function in $\mathcal{E}\left(\mathbb{C}^{+}\right)$, and by Corollary 2.8 the function

$$
\zeta \mapsto g_{j k}\left(i \frac{1+\zeta}{1-\zeta}\right)
$$

extends from $S$ to a holomorphic function in $\mathcal{A}_{*}^{\infty}(\bar{\Delta})$ flat at 1 . Hence the same is true for the function

$$
\zeta \mapsto z_{2}(\zeta)=2 i \sum_{\substack{j>k \\ 2 \leq j+k \leq m}} a_{j k} g_{j k}\left(i \frac{1+\zeta}{1-\zeta}\right) .
$$

Therefore the disc $A(\zeta)=\left(z_{1}(\zeta), z_{2}(\zeta)\right)$ is flat at 1 by Lemma 3.6 and Corollary 2.8. It remains to show that $A(\zeta)$ is attached to $M$ through 0 , i.e. $\Im z_{2}(\zeta)=p\left(z_{1}(\zeta), \bar{z}_{1}(\zeta)\right), \zeta \in S$, which follows from (3.2) and the hypothesis $a_{j k}=0$ for $j=k$. This proves Theorem 2 . 


\section{Remarks}

The following is proved easily from Theorem 1 .

Proposition 4.1. Let $M$ be a hypersurface in $\mathbb{C}^{n+1}$ given by

$$
\begin{aligned}
\left\{\left(z^{\prime}, z_{n+1}\right)\right. & \left.\in \mathbb{C}^{n+1}: \Im z_{n+1}=p\left(z^{\prime}, \bar{z}^{\prime}\right)\right\} \\
p\left(z^{\prime}, \bar{z}^{\prime}\right) & =\sum_{2 \leq|\alpha|+|\beta| \leq m} a_{\alpha \beta} z^{\prime \alpha} \bar{z}^{\prime} \beta
\end{aligned}
$$

with $a_{\alpha \beta}=\bar{a}_{\beta \alpha}$. If $a_{\alpha \beta}=0$ when $|\alpha|+|\beta|$ is even, then given $\epsilon>0$ there are nontrivial smooth analytic discs $A$ attached to $M$ through the origin, flat at 1 , and satisfying $|A(\zeta)|<\epsilon$ for all $\zeta \in \bar{\Delta}$.

Proof. For $n=1$, the proposition is a special case of Theorem 1. For $n>1$, put $z_{j}(\zeta) \equiv 0$ for $j=2, \ldots, n$ and define $z_{1}(\zeta)$ by (3.5) of Theorem 2 . Following the proof of Theorem 2, it is not hard to check that the disc so determined satisfies the desired conclusion.

Remark 4.3. In contrast with Proposition 4.1, note that if $M$ is a hypersurface in $\mathbb{C}^{n+1}, 0 \in M$, and $M$ strictly pseudoconvex, then any small disc $A$ attached to $M$ through 0 has the property that $A^{\prime}(1)$ is not tangent to $M$ (and hence $A^{\prime}(1) \neq 0$ ). This is well known and can be shown by the following argument. After a change of holomorphic coordinates near $0, M$ is given by

$$
\Im z_{n+1}=\phi\left(z^{\prime}, \Re z_{n+1}\right),
$$

with $\phi(0)=0$ and $\phi\left(z^{\prime}, \Re z_{n+1}\right) \geq C\left(\left|z^{\prime}\right|^{2}+\left|\Re z_{n+1}\right|^{2}\right)$ near 0 , with $C>0$. The nontangency claimed above is then equivalent to the strict inequality

$$
\int_{0}^{2 \pi} \frac{\phi\left(z^{\prime}\left(e^{i \theta}\right), \Re z_{n+1}\left(e^{i \theta}\right)\right)}{\left|e^{i \theta}-1\right|^{2}} d \theta>0
$$

for $A(\zeta)=\left(z^{\prime}(\zeta), z_{n+1}(\zeta)\right)$, which holds, taking into account the properties of $\phi$, since $A$ is nonconstant.

Remark 4.4. Let $M$ be a homogeneous hypersurface in $\mathbb{C}^{2}$, i.e. $M$ given by $\Im z_{2}=p\left(z_{1}, \bar{z}_{1}\right)$ with $p$ a real valued homogeneous polynomial. As in [2], we shall say that $M$ is minimally convex at 0 if $p$ is not harmonic and if either

$$
\int_{0}^{2 \pi} \frac{p\left(z_{1}\left(e^{i \theta}\right), \overline{z_{1}\left(e^{i \theta}\right)}\right)}{\left|e^{i \theta}-1\right|^{2}} d \theta \geq 0
$$

for all smooth analytic discs $z_{1}(\cdot) \in \mathcal{A}_{*}^{\infty}(\Delta)$, or if the left hand side of (4.3) is nonpositive for all such discs. The following generalization of 
Remark 4.3 is proved in Corollary (5.16) in [3]. If $M \subset \mathbb{C}^{2}$ is homogeneous and minimally convex at 0 and if $A(\zeta)$ is a smooth nonconstant analytic disc attached to $M$ through 0 , then $A^{\prime}(1)$ is not tangent to $M$. (In particular, $A^{\prime}(1) \neq 0$.)

Remark 4.6. It is shown in Huang-Krantz [7] that if $A$ is a nonconstant smooth analytic disc partially attached to a strictly pseudoconvex hypersurface in $\mathbb{C}^{n+1}$, then $A$ cannot be flat at 1 .

\section{References}

1. S. Alinhac, M. S. Baouendi, and L. P. Rothschild, Unique continuation and regularity at the boundary for holomorphic functions, Duke J. Math. 61 (1990), 635-653.

2. M. S. Baouendi and L. P. Rothschild, A generalized complex Hopf Lemma and its applications to CR mappings, Invent. Math. 111 (1993), 331-348.

3. M. S. Baouendi, L. P. Rothschild, and J.-P. Trépreau, On the geometry of discs attached to real manifolds, J. Diff. Geom., to appear.

4. L. Boutet de Monvel, Boundary problems for pseudodifferential operators, Acta Math. 126 (1971), 11-51.

5. E. Bishop, Differentiable manifolds in complex Euclidean space, Duke Math. J. 32 (1965), 1-22.

6. L. Hörmander, The analysis of partial differential operators I, Springer, Berlin, 1983.

7. S. Huang and S. G. Krantz, A unique continuation problem for holomorphic mappings, Comm. P.D.E. 18 (1993), 241-263.

8. J. J. Kohn, Boundary behavior of $\bar{\partial}$ on weakly pseudoconvex manifolds of dimension two, J. Diff. Geom. 6 (1972), 523-542.

9. H. Lewy, On the local character of the solution of an atypical differential equation in three variables and a related problem for regular functions of two complex variables, Ann. of Math. 64 (1956), 514-522.

10. J.-M. Trépreau, Sur le prolongement holomorphe des fonctions $C R$ définies sur une hypersurface réelle de classe $C^{2}$ dans $\mathbb{C}^{n}$, Invent. Math. 83 (1986), 583-592.

11. A. E. Tumanov, Extension of $C R$ functions into a wedge from a manifold of finite type, Mat. Sbornik 136 (1988), 128-139; English trans. in Math. USSR Sbornik 64 (1989), 129-140.

Universite de Paris-Sud, Departement de Mathematique, 91405 Orsay, France. E-mail address: salinhac@matups.matups.fr

Department of Mathematics-0112, University of California, San Diego, La JollA, CA 92093-0112.

E-mail address: sbaouendi@ucsd.edu, lrothschild@ucsd.edu 